\title{
Disaggregation of bipolar-valued outranking relations
}

\author{
Patrick Meyer $^{1}$, Jean-Luc Marichal ${ }^{2}$, and Raymond Bisdorff ${ }^{2}$ \\ 1 Institut TELECOM, TELECOM Bretagne, LabSTICC - UMR 3192, \\ Technople Brest-Iroise CS 83818, F-29238 Brest Cedex 3, FRANCE, \\ patrick.meyer@telecom-bretagne.eu \\ 2 University of Luxembourg, 162a, avenue de la Faïencerie, L-1511 Luxembourg, \\ \{raymond.bisdorff, jean-luc.marichal\}@uni.lu
}

\begin{abstract}
In this article, we tackle the problem of exploring the structure of the data which is underlying a bipolar-valued outranking relation. More precisely, we show how the performances of alternatives and weights related to criteria can be determined from three different formulations of the bipolar-valued outranking relations, which are given beforehand.
\end{abstract}

Key words: bipolar-valued outranking relation, disaggregation, mixed integer programming

\section{Introduction}

Let $X=\{x, y, z, \ldots\}$ be a set of $p$ alternatives and $N=\{1, \ldots, n\}$ be a set of $n$ criteria. Each alternative of $X$ is evaluated on each of the criteria of $N$. Let us write $g_{i}(x)$ for the performance of alternative $x$ on criterion $i$ of $N$. In this work, we will regard, without any loss of generality, such a performance function $g_{i}(i \in N)$ as having its values in $[0,1]$ s.t.:

$$
\forall x, y \in X, g_{i}(x) \geq g_{i}(y) \Rightarrow x \text { is at least as good as } y \text { on criterion } i \text {. }
$$

With each criterion $i$ of $N$ we associate its weight represented by a rational number $w_{i}$ from the interval $[0,1]$ such that

$$
\sum_{i=1}^{n} w_{i}=1 .
$$

To enrich the model which can be based on Formula (1), it is possible to associate different thresholds (weak preference, preference, weak veto, veto; see, e.g., [BMR07]) with the criteria functions which allow to represent more precisely a decision maker's (DM's) local "at least as good as" preferences.

Let $S$ be a binary relation on $X$. Classically, the proposition " $x$ outranks $y$ " $(x S y)(x, y \in X)$ is assumed to be validated if there is a sufficient majority of criteria which supports an "at least as good as" preferential statement and there is no criterion which raises a veto against it [Roy85]. 
In this paper, given the outranking relation, we detail how the performances of the alternatives and the weights associated with the criteria can be determined. We present three different definitions of the outranking relation, where the first model takes only into account a preference threshold, the second one considers also a weak preference threshold, and finally, the third one adds also two veto thresholds.

From a practical point of view, the determination of the performances of the alternatives on the criteria may be questionnable, as in general, in a decision problem, these evaluations are given beforehand. Nevertheless, from an experimental point of view, the determination of a performance table from a given valued outranking relation can be of some help. Furthermore, it is possible to show that our developments can easily be extended to the tuning of the parameters underlying the DM's preferences.

\section{$2 \mathcal{M}_{1}$ : Model with a single preference threshold}

Starting from Formula (1), this first model enriches the local pairwise comparison of two alternatives on each criterion by a preference threshold. Therefore, to characterise a local "at least as good as" situation between two alternatives $x$ and $y$ of $X$, for each criterion $i$ of $N$, we use the function $C_{i}: X \times X \rightarrow\{-1,1\}$ defined by:

$$
C_{i}(x, y)=\left\{\begin{aligned}
1 & \text { if } g_{i}(y)<g_{i}(x)+p \\
-1 & \text { otherwise }
\end{aligned}\right.
$$

where $p \in] 0,1[$ is a constant preference threshold associated with all the preference dimensions. According to this local concordance index, $x$ is considered as at least as good as $y$ for criterion $i$ if $g_{i}(y)<g_{i}(x)+p\left(C_{i}(x, y)=1\right)$. Else, $x$ is not considered as at least as good as $y$ for criterion $i\left(C_{i}(x, y)=-1\right)$.

The overall outranking index $\widetilde{S}$, defined for all pairs of alternatives $(x, y) \in$ $X \times X$, can then be written as:

$$
\widetilde{S}(x, y)=\sum_{i \in N} w_{i} C_{i}(x, y)
$$

$\widetilde{S}$ represents the credibility of the validation or non-validation of an outranking situation observed between each pair of alternatives [BMR07]. The maximum value 1 of $\widetilde{S}$ is reached in the case of unanimous concordance, whereas the minimum value -1 is obtained in the case of unanimous discordance. $\widetilde{S}$ is called the bipolar-valued characterisation of the outranking relation $S$, or, for short, the bipolar-valued outranking relation.

Given the bipolar-valued outranking relation $\widetilde{S}$ and a constant preference threshold $p$, we now show how the values taken by the performance functions $g_{i}(x)(\forall i \in N, \forall x \in X)$ and the associated weights $w_{i}(\forall i \in N)$ can be determined.

The local concordance conditions (2) can be translated as follows into linear constraints: 
$(-1+p)\left(C_{i}(x, y)-1\right)<g_{i}(x)-g_{i}(y)+p \leq(1+p)\left(C_{i}(x, y)+1\right) \quad \forall x \neq y \in X, \forall i \in N$,

where $C_{i}(x, y) \in\{-1,1\}$ for each $x \neq y \in X$. Indeed, $g_{i}(x)-g_{i}(y)+p>0$ implies $C_{i}(x, y)=1$ whereas $g_{i}(x)-g_{i}(y)+p \leq 0$ forces $C_{i}(x, y)=-1$.

Constraints derived from Equation 3 can be written as

$$
\sum_{i=1}^{n} w_{i}^{\prime}(x, y)=\widetilde{S}(x, y) \quad \forall x \neq y \in X,
$$

where $w_{i}^{\prime}(x, y)$ is a non-negative variable for each $i \in N, x \neq y \in X$ s.t.:

$$
w_{i}^{\prime}(x, y)=\left\{\begin{aligned}
w_{i} & \text { if } C_{i}(x, y)=1 \\
-w_{i} & \text { otherwise }
\end{aligned}\right.
$$

This then leads to the following linear constraints:

$$
\begin{aligned}
& -w_{i} \leq w_{i}^{\prime}(x, y) \leq w_{i} \\
& w_{i}+C_{i}(x, y)-1 \leq w_{i}^{\prime}(x, y) \\
& w_{i}^{\prime}(x, y) \leq-w_{i}+C_{i}(x, y)+1
\end{aligned}
$$

Indeed, $C_{i}(x, y)=-1$ implies $w_{i}^{\prime}(x, y)=-w_{i}$, whereas $C_{i}(x, y)=1$ forces $w_{i}^{\prime}(x, y)=w_{i}$.

In order to remain flexible enough and not to depend on rounding errors, we propose to approach the values taken by $\widetilde{S}$ as closely as possible by minimising the maximal gap between $\widetilde{S}(x, y)$ and $\sum_{i \in N} w_{i} C_{i}(x, y)$, for all $x \neq y \in X$, represented by a non-negative variable $\varepsilon$.

The mixed integer program MIP1 which has to be solved can now be written as follows:

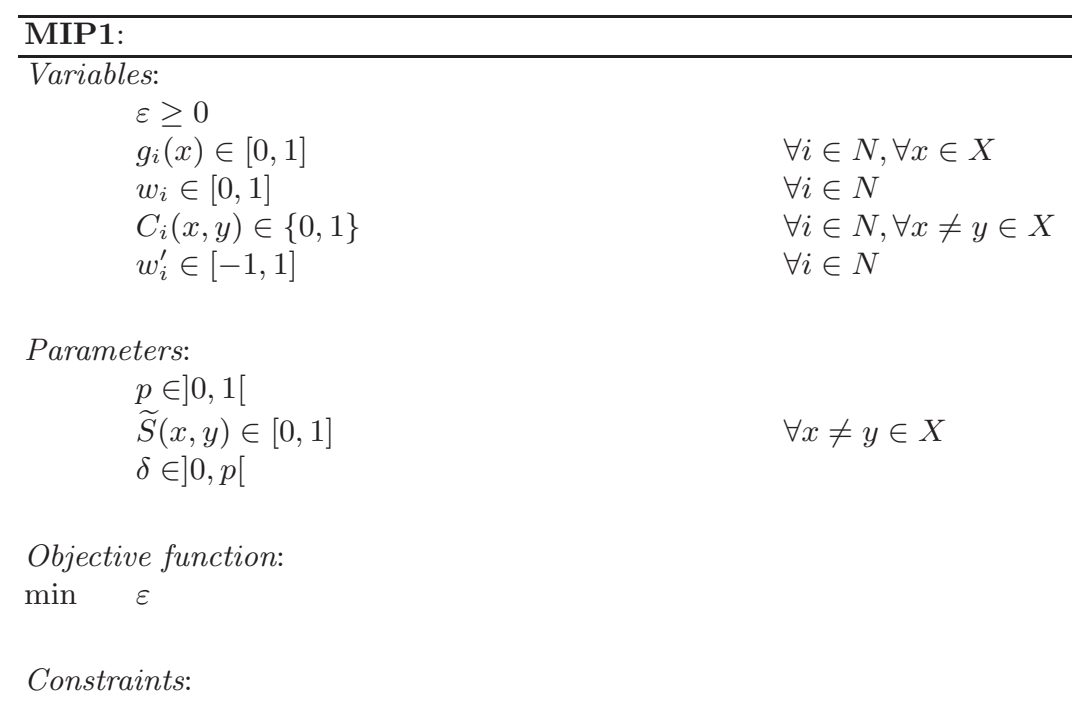




$$
\begin{aligned}
& \text { s.t. } \quad \sum_{i=1}^{n} w_{i}=1 \\
& -w_{i} \leq w_{i}^{\prime}(x, y) \quad \forall x \neq y \in X, \forall i \in N \\
& w_{i}^{\prime}(x, y) \leq w_{i} \quad \forall x \neq y \in X, \forall i \in N \\
& w_{i}+C_{i}(x, y)-1 \leq w_{i}^{\prime}(x, y) \quad \forall x \neq y \in X, \forall i \in N \\
& w_{i}^{\prime}(x, y) \leq-w_{i}+C_{i}(x, y)+1 \quad \forall x \neq y \in X, \forall i \in N \\
& \sum_{i=1}^{n} w_{i}^{\prime}(x, y) \leq \widetilde{S}(x, y)+\varepsilon \quad \forall x \neq y \in X \\
& \sum_{i=1}^{i=1} w_{i}^{\prime}(x, y) \geq \widetilde{S}(x, y)-\varepsilon \quad \forall x \neq y \in X \\
& (-1+p)\left(1-C_{i}(x, y)\right)+\delta \leq g_{i}(x)-g_{i}(y)+p \forall x \neq y \in X, \forall i \in N \\
& g_{i}(x)-g_{i}(y)+p \leq(1+p) C_{i}(x, y) \quad \forall x \neq y \in X, \forall i \in N
\end{aligned}
$$

The solution of MIP1 might not be unique. If the objective function equals 0 , then there exist $g_{i}(x)(\forall i \in N, \forall x \in X)$ and associated weights $w_{i}(\forall i \in N)$ generating the overall outranking index $\widetilde{S}$ via Equations (2) and (3). Else there exists no solution to the problem via the selected representation, and the output of MIP1 can be considered as an approximation of the given $\widetilde{S}$ by a the constant preference threshold model.

Let us now turn to a more complex model which allows to represent a larger set of valued outranking relations.

\section{$3 \mathcal{M}_{2}$ : Model with two preference thresholds}

In this case, a local "at least as good as" situation between two alternatives $x$ and $y$ of $X$ is characterised by the function $C_{i}^{\prime}: X \times X \rightarrow\{-1,0,1\}$ s.t.:

$$
C_{i}^{\prime}(x, y)=\left\{\begin{aligned}
1 & \text { if } g_{i}(y)<g_{i}(x)+q \\
-1 & \text { if } g_{i}(y) \geq g_{i}(x)+p \\
0 & \text { otherwise }
\end{aligned}\right.
$$

where $q \in] 0, p$ [ is a constant weak preference threshold associated with all the preference dimensions. If $C_{i}^{\prime}(x, y)=1\left(\operatorname{resp} . C_{i}^{\prime}(x, y)=-1\right)$, then $x$ is considered (resp. not considered) as at least as good as $y$ for criterion $i$. Finally, according to the developments in [BMR07], if $g_{i}(x)+q \leq g_{i}(y)<g_{i}(x)+p$ then it cannot be determined whether $x$ is at least as good as $y$ or not for criterion $i$, and $C_{i}^{\prime}(x, y)=0$.

The overall outranking index $\widetilde{S}^{\prime}$ is defined as follows for all pairs of alternatives $(x, y) \in X \times X$ :

$$
\widetilde{S}^{\prime}(x, y)=\sum_{i \in N} w_{i} C_{i}^{\prime}(x, y) .
$$

According to Equation (6), $\widetilde{S}^{\prime}$ has its values in $[-1,1]$. Its maximum value 1 is reached in the case of unanimous concordance, its minimum value -1 represents unanimous discordance, and the value 0 is obtained if the positive arguments counterbalance the negative arguments for the outranking. The value 0 therefore 
represents an indetermined outranking situation. In this context, $\widetilde{S}^{\prime}$ is again called the bipolar-valued outranking relation.

In order to represent the three values taken by $C_{i}^{\prime}(x, y)$, we use two binary variables $\alpha_{i}(x, y) \in\{0,1\}$ and $\beta_{i}(x, y) \in\{0,1\}(\forall i \in N, \forall x \neq y \in X)$ s.t.

$$
C_{i}^{\prime}(x, y)=\alpha_{i}(x, y)-\beta_{i}(x, y) .
$$

Note that $C_{i}^{\prime}(x, y)=1$ if $\alpha_{i}(x, y)=1$ and $\beta_{i}(x, y)=0, C_{i}^{\prime}(x, y)=-1$ if $\alpha_{i}(x, y)=0$ and $\beta_{i}(x, y)=1$, and $C_{i}^{\prime}(x, y)=0$ if $\alpha_{i}(x, y)=\beta_{i}(x, y)=1$ or $\alpha_{i}(x, y)=\beta_{i}(x, y)=0$.

The local concordance conditions (5) can then be rewritten as follows as linear constraints $(\forall x \neq y \in X, \forall i \in N)$ :

$$
\left\{\begin{aligned}
(-1+q)\left(1-\alpha_{i}(x, y)\right) & <g_{i}(x)-g_{i}(y)+q \leq(1+q) \alpha_{i}(x, y) ; \\
(-1+p) \beta_{i}(x, y) & <g_{i}(x)-g_{i}(y)+p \leq(1+p)\left(1-\beta_{i}(x, y)\right) .
\end{aligned}\right.
$$

Note that, as $p>q>0, g_{i}(x)-g_{i}(y)+q>0 \Rightarrow g_{i}(x)-g_{i}(y)+p>0$, and $g_{i}(x)-g_{i}(y)+p<0 \Rightarrow g_{i}(x)-g_{i}(y)+q<0$. Consequently, in constraints (8), $g_{i}(x)-g_{i}(y)+q>0$ forces $\alpha_{i}(x, y)=1$ and $\beta_{i}(x, y)=0\left(C_{i}^{\prime}(x, y)=1\right)$ whereas $g_{i}(x)-g_{i}(y)+p<0$ implies $\beta_{i}(x, y)=1$ and $\alpha_{i}(x, y)=0\left(C_{i}^{\prime}(x, y)=-1\right)$. Furthermore, $g_{i}(x)-g_{i}(y)+q<0$ and $g_{i}(x)-g_{i}(y)+p>0$ implies $\alpha_{i}(x, y)=$ $\beta_{i}(x, y)=0\left(C_{i}^{\prime}(x, y)=0\right)$. Then, $g_{i}(x)-g_{i}(y)+q=0 \Rightarrow g_{i}(x)-g_{i}(y)+p>0$ forces $\alpha_{i}(x, y)=\beta_{i}(x, y)=0$ and finally $g_{i}(x)-g_{i}(y)+p=0 \Rightarrow g_{i}(x)-g_{i}(y)+q<$ 0 implies that $\alpha_{i}(x, y)=0$ and $\beta_{i}(x, y)=1\left(C_{i}^{\prime}(x, y)=1\right)$.

It is important to note that constraints (8) linked to the condition $p>q>0$ do not allow that $\alpha_{i}(x, y)=\beta_{i}(x, y)=1$ simultanously. Indeed $\alpha_{i}(x, y)=1 \Rightarrow$ $g_{i}(x)-g_{i}(y)+q \geq 0$ and $\beta_{i}(x, y)=1 \Rightarrow g_{i}(x)-g_{i}(y)+p \leq 0$, which is only possible if $p=q$.

Equation (6) can be rewritten as follows:

$$
\widetilde{S}^{\prime}(x, y)=\sum_{i \in N} w_{i}\left(\alpha_{i}(x, y)-\beta_{i}(x, y)\right) \quad \forall x \neq y \in X,
$$

which can be replaced by a linear constraint of the type

$$
\sum_{i=1}^{n} w_{i}^{\prime \prime}(x, y)=\widetilde{S}^{\prime}(x, y) \quad \forall x \neq y \in X,
$$

where $w_{i}^{\prime \prime}(x, y) \in[-1,1]$ for each $i \in N, x \neq y \in X$ s.t.:

$$
w_{i}^{\prime \prime}(x, y)=\left\{\begin{array}{c}
w_{i} \text { if } C_{i}^{\prime}(x, y)=1 ; \\
-w_{i} \text { if } C_{i}^{\prime}(x, y)=-1 ; \\
0 \text { otherwise. }
\end{array}\right.
$$

This then leads to the following linear constraints $(\forall x \neq y \in X, \forall i \in N)$ :

$$
\begin{aligned}
& -w_{i} \leq w_{i}^{\prime \prime}(x, y) \leq w_{i} \\
& w_{i}+\alpha_{i}(x, y)-\beta_{i}(x, y)-1 \leq w_{i}^{\prime \prime}(x, y) \\
& w_{i}^{\prime \prime}(x, y) \leq-w_{i}+\alpha_{i}(x, y)-\beta_{i}(x, y)+1 \\
& -\left[\alpha_{i}(x, y)+\beta_{i}(x, y)\right] \leq w_{i}^{\prime \prime}(x, y) \leq \alpha_{i}(x, y)+\beta_{i}(x, y) .
\end{aligned}
$$


Indeed, recalling that $\alpha_{i}(x, y)$ and $\beta_{i}(x, y)$ cannot simultanously be equal to 1 , it is easy to verify that $C_{i}^{\prime}(x, y)=1 \Rightarrow w_{i}^{\prime \prime}(x, y)=w_{i}, C_{i}^{\prime}(x, y)=-1 \Rightarrow w_{i}^{\prime \prime}(x, y)=$ $-w_{i}$, and $C_{i}^{\prime}(x, y)=0 \Rightarrow w_{i}^{\prime \prime}(x, y)=0$.

These considerations lead to the formulation of the mixed integer program MIP2, whose objective is again to minimise a non-negative variable $\varepsilon$ representing the maximal gap between $\widetilde{S}^{\prime}(x, y)$ and $\sum_{i \in N} w_{i} C_{i}^{\prime}(x, y)$, for all $x \neq y \in X$.

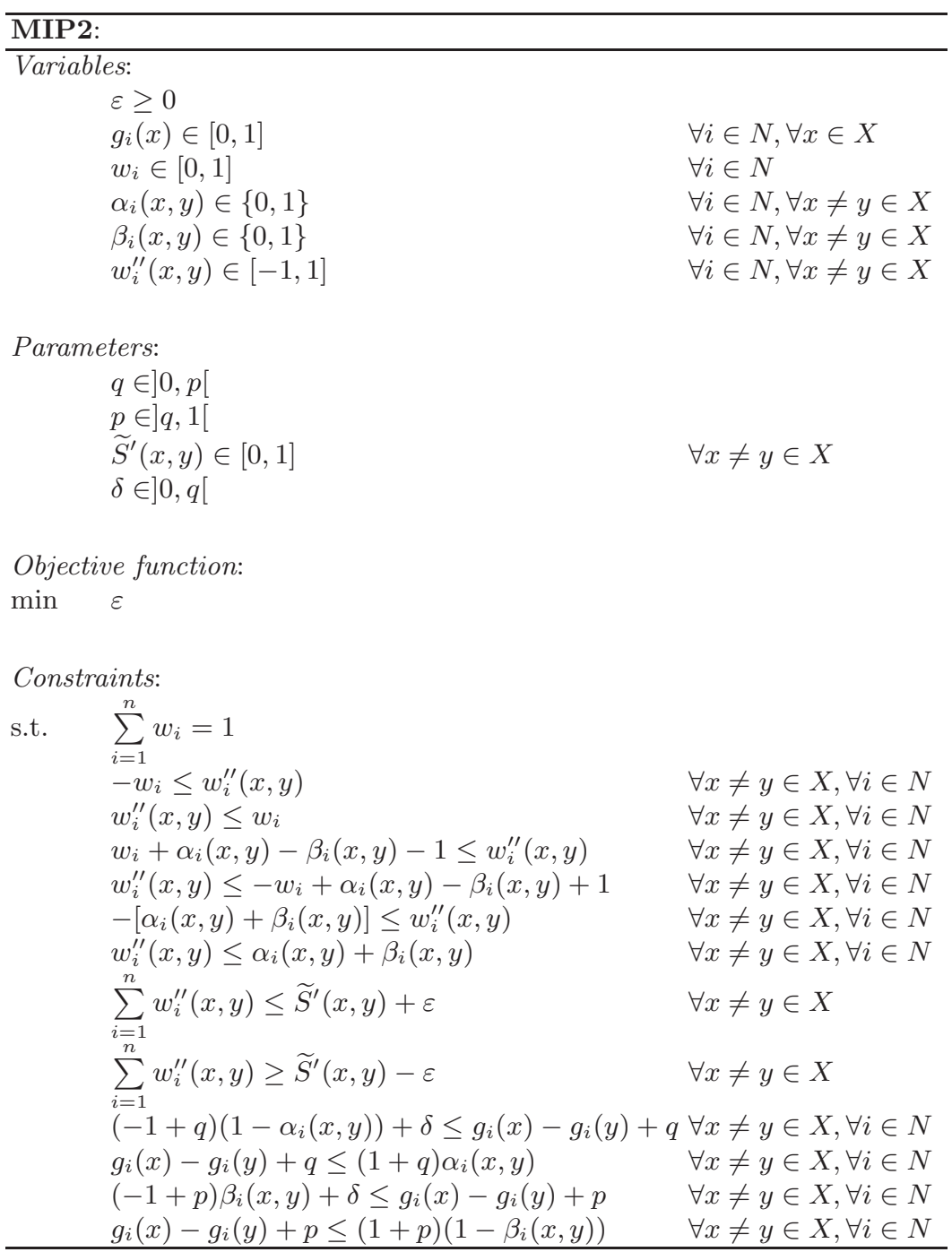

Similar remarks as for MIP1 concerning the uniqueness and the characteristics of the solution apply here. Once again, let us now turn to a more complex model which allows to represent an even larger set of valued outranking relations. 


\section{$4 \mathcal{M}_{3}$ : Model with two preference and two veto thresholds}

In this third case, the outranking relation is enriched by veto thresholds on the criteria. A veto threshold on a criterion $i \in N$ allows to clearly non-validate an outranking situation between two alternatives if the difference of evaluations on $i$ is too large. A local veto situation for each criterion $i$ of $N$ is characterised by a veto function $V_{i}: X \times X \rightarrow\{-1,0,1\}$ s.t.:

$$
V_{i}(x, y)=\left\{\begin{aligned}
1 & \text { if } g_{i}(y) \geq g_{i}(x)+v \\
-1 & \text { if } g_{i}(y)<g_{i}(x)+w v \\
0 & \text { otherwise }
\end{aligned}\right.
$$

where $w v \in] p, 1[$ (resp. $v \in] w v, 1[$ ) is a constant weak veto threshold (resp. veto threshold) associated with all the preference dimensions. If $V_{i}(x, y)=1$ (resp. $V_{i}(x, y)=-1$ ), then the comparison of $x$ and $y$ for criterion $i$ leads (resp. does not lead) to a veto. Again, according to the developments in [BMR07], if $g_{i}(x)+w v<g_{i}(y) \leq g_{i}(x)+v$ then it cannot be determined whether we have a veto situation between $x$ and $y$ or not, and $V_{i}(x, y)=0$. Figure 1 represents the local concordance and veto indexes for a fixed $g_{i}(x)$.

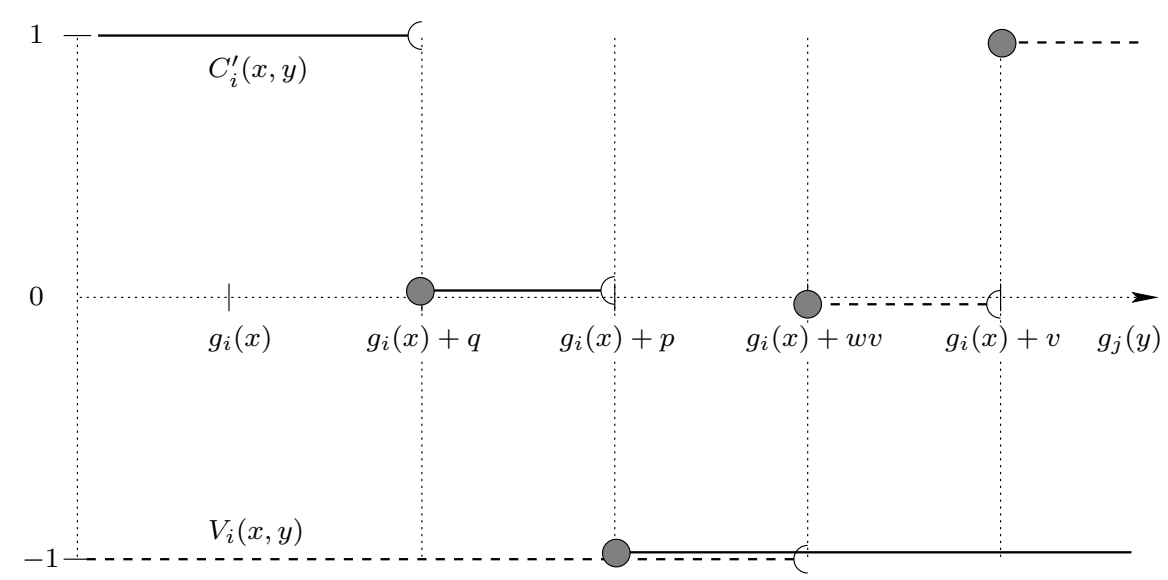

Fig. 1. Local concordance and veto indexes for a fixed $g_{i}(x)$

To take into account these veto effects, the overall outranking index $\widetilde{S}^{\prime \prime}$ is defined as follows for all pairs of alternatives $(x, y) \in X \times X$ :

$$
\widetilde{S}^{\prime \prime}(x, y)=\min \left\{\sum_{i \in N} w_{i} C_{i}^{\prime}(x, y),-V_{1}(x, y), \ldots,-V_{n}(x, y)\right\} .
$$

The min operator in Formula (10) tranlsates the conjunction between the overall concordance and the negated local veto indexes for each criterion. In the case of absence of veto on all the criteria $\left(V_{i}=-1 \forall i \in N\right)$, we have $\widetilde{S}^{\prime \prime}(x, y)=\widetilde{S}^{\prime}(x, y)$. 
Similarly as in Section 3, the three values taken by the local veto function can be represented by means of two binary variables $\alpha_{i}^{\prime}(x, y) \in\{0,1\}$ and $\beta_{i}^{\prime}(x, y) \in$ $\{0,1\}(\forall i \in N, \forall x \neq y \in X)$ s.t.

$$
V_{i}(x, y)=\alpha_{i}^{\prime}(x, y)-\beta_{i}^{\prime}(x, y) .
$$

Recalling that $w v<v$, conditions (9) can then be rewritten as follows as linear constraints $(\forall x \neq y \in X, \forall i \in N)$ :

$$
\left\{\begin{aligned}
(-1+w v)\left(1-\beta_{i}^{\prime}(x, y)\right) & <g_{i}(x)-g_{i}(y)+w v \leq(1+w v) \beta_{i}^{\prime}(x, y) \\
(-1+v) \alpha_{i}^{\prime}(x, y) & <g_{i}(x)-g_{i}(y)+v \leq(1+v)\left(1-\alpha_{i}^{\prime}(x, y)\right) .
\end{aligned}\right.
$$

To represent Formula (10) as a set of linear constraints, we need to introduce some further binary variables $z_{0}(x, y)$ and $z_{i}(x, y)(\forall x \neq y \in X, \forall i \in N)$ s.t.:

$\widetilde{S}^{\prime \prime}(x, y)=\left\{\begin{aligned}-V_{k}(x, y) & \text { if } \quad z_{k}(x, y)=1 \text { and } z_{i}(x, y)=0 \quad \forall i \in N \cup\{0\} \backslash\{k\} ; \\ \sum_{i \in N} w_{i} C_{i}^{\prime}(x, y) & \text { if } \quad z_{0}(x, y)=1 \text { and } z_{i}(x, y)=0 \quad \forall i \in N .\end{aligned}\right.$

This leads to the following linear constraints:

$$
\begin{array}{ll}
\widetilde{S}^{\prime \prime}(x, y) \leq \sum_{i \in N} w_{i} C_{i}^{\prime}(x, y) & \forall x \neq y \in X ; \\
\widetilde{S}^{\prime \prime}(x, y) \leq-\left(\alpha_{i}^{\prime}(x, y)-\beta_{i}^{\prime}(x, y)\right) & \forall x \neq y \in X, \forall i \in N ; \\
\sum_{i \in N} w_{i} C_{i}^{\prime}(x, y) \leq 2\left(1-z_{0}(x, y)\right)+\widetilde{S}^{\prime \prime}(x, y) & \forall x \neq y \in X ; \\
-\left(\alpha_{i}^{\prime}(x, y)-\beta_{i}^{\prime}(x, y)\right) \leq 2\left(1-z_{i}(x, y)\right)+\widetilde{S}^{\prime \prime}(x, y) & \forall x \neq y \in X, \forall i \in N ; \\
\sum_{i=0}^{n} z_{i}(x, y)=1 & \forall x \neq y \in X .
\end{array}
$$

Due to the last condition of Constraints (12), there exists a unique $k \in N \cup\{0\}$ s.t. $z_{k}=1$ and $z_{i}=0$ for $i \in N \cup\{0\} \backslash\{k\}$. Besides, if $\sum_{i \in N} w_{i} C_{i}^{\prime}(x, y)<-V_{i}(x, y)$ holds for all $i \in N$, then $z_{i}(x, y)=0$ for all $i \in N$ and $z_{0}(x, y)=1$ (which implies that $\left.\widetilde{S}^{\prime \prime}(x, y)=\sum_{i \in N} w_{i} C_{i}^{\prime}(x, y)\right)$. Furthermore, if $\exists k \in N \cup\{0\}$ s.t. $-V_{k}(x, y)<$ $\sum_{i \in N} w_{i} C_{i}^{\prime}(x, y)$ and $-V_{k}(x, y)<-V_{i}(x, y)(\forall i \in N \backslash\{k\})$, then $z_{k}(x, y)=1$ (which implies that $\widetilde{S}^{\prime \prime}(x, y)=-V_{k}(x, y)$ ).

Constraints (12) only represent Formula (10) if all criteria have strictly positive weights. Note also that the first and the third condition of Constraints (12) can easily be linearised as in Section 3 .

These considerations lead to the formulation of the mixed integer program MIP3, whose objective is to minimise a non-negative variable $\varepsilon$ representing the maximal gap between $\widetilde{S}^{\prime \prime}(x, y)$ and $\sum_{i \in N} w_{i} C_{i}^{\prime}(x, y)$, for all $x \neq y \in X$ where the bipolar-valued outranking relation requires no veto. As $\widetilde{S}^{\prime \prime}(x, y)$ equals -1 or 0 in veto situations, no gap is considered on these values. Remember that all the weights are supposed to be strictly positive. 


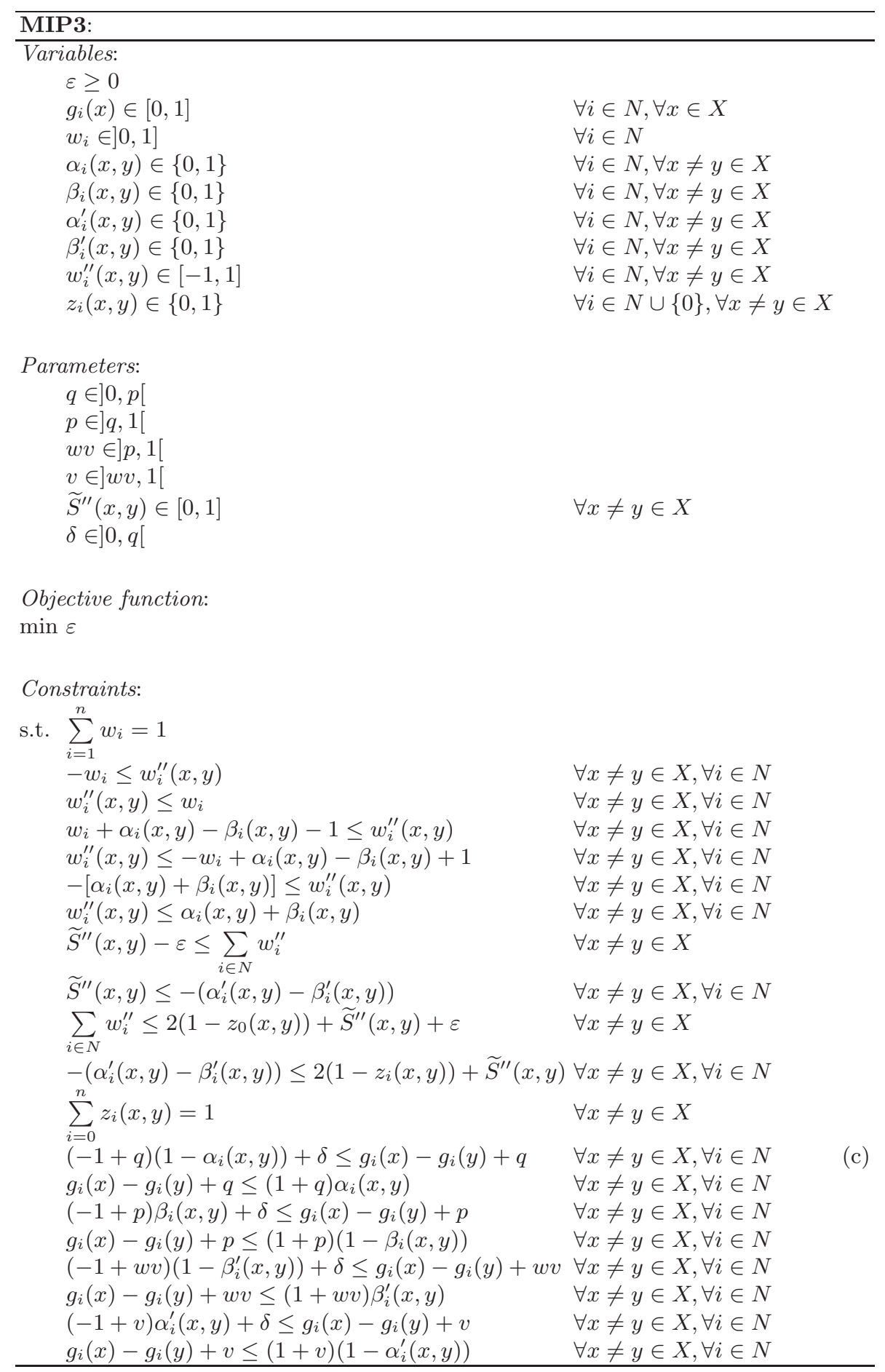




\subsection{Example}

Let us consider the bipolar-valued outranking relation $\widetilde{S}$ on $X=\{a, b, c\}$ of Table 1 and fix $q=0.1, p=0.2, w v=0.6$ and $v=0.8$. Let us first try to

\begin{tabular}{c|ccc}
\hline$\widetilde{S}$ & $a$ & $b$ & $c$ \\
\hline$a$ & $\cdot$ & 0.258 & -0.186 \\
$b$ & 0.334 & $\cdot$ & 0.556 \\
$c$ & -1.000 & 0.036 & $\cdot$ \\
\hline
\end{tabular}

Table 1. Given $\widetilde{S}$

\begin{tabular}{|c|c|c|c|c|c|}
\hline$\widetilde{S}^{*}$ & $a$ & $b$ & $c$ & $g_{1}$ & $g_{2}$ \\
\hline$a$ & & 0.407 & 0.407 & 0.280 & 0.000 \\
\hline$b$ & 0.296 & . & 1.000 & 0.090 & 1.000 \\
\hline$c$ & -0.407 & 0.407 & . & 0.000 & 0.200 \\
\hline$\overline{w_{i}}$ & & & & 0.704 & 0.296 \\
\hline
\end{tabular}

Table 2. Approximative outranking relation $\widetilde{S}^{*}$ via MIP1bis for $n=4$

represent $\widetilde{S}$ by model $\mathcal{M}_{2}$. For $n=4$, the value of the objective function for the optimal solution of MIP2 equals 0.593 . The weights $w_{3}$ and $w_{4}$ equal 0 . Table 2 summarises the outranking relation associated with its optimal solution determined by solving MIP2 for $n=4$. One can easily check that $\widetilde{S}$ and $\widetilde{S}^{*}$ differ by at most 0.593 . This shows that this outranking relation is not representable by $\mathcal{M}_{2}$ and at most 4 criteria. We therefore switch to the more general model $\mathcal{M}_{3}$ with two preference and two veto thresholds.

For $n=4$ the value of the objective function for the optimal solution of MIP3 equals 0 . This means that $\widetilde{S}$ can be built from a performance table with 4 criteria via $\mathcal{M}_{3}$, given the above thresholds. For lower values of $n$, the objective function for the optimal solution is strictly positive. Table 3 shows the performances of the three alternatives and the weights which allow to construct $\widetilde{S}$ via model $\mathcal{M}_{3}$. A veto situation occurs between $a$ and $c$ on criterion $g_{4}(\widetilde{S}(c, a)=-1)$.

\begin{tabular}{r|cccc}
\hline & $g_{1}$ & $g_{2}$ & $g_{3}$ & $g_{4}$ \\
\hline$a$ & 0.000 & 0.000 & 0.000 & 1.000 \\
$b$ & 0.400 & 0.100 & 0.090 & 0.590 \\
$c$ & 0.200 & 0.290 & 0.000 & 0.000 \\
\hline$w_{i}$ & 0.149 & 0.444 & 0.074 & 0.333 \\
\hline
\end{tabular}

Table 3. Performances and weights to construct $\widetilde{S}$ via model $\mathcal{M}_{3}$

\section{References}

[BMR07] R. Bisdorff, P. Meyer, and M. Roubens. Rubis: a bipolar-valued outranking method for the best choice decision problem. 4OR, Quaterly Journal of the Belgian, French and Italian Operations Research Societies, 2007. in press, doi:10.1007/s10288-007-0045-5.

[Roy85] B. Roy. Méthodologie multicritère d'aide à la décision. Ed. Economica, collection Gestion, 1985. 